\title{
Spinal stimulation and physical therapy helps paraplegic patients to walk again
}

Overground walking can be restored in people with complete loss of motor function in the lower limbs, according to two new studies published in the New England Journal of Medicine (NEJM) and Nature Medicine. Epidural electrical stimulation of the spinal cord in combination with physical therapy enabled patients to sit, stand and walk.

Research in animal models of spinal cord injury (SCI) has shown that electrical stimulation from a device implanted into the spinal epidural space can restore standing and stepping. In humans, epidural stimulation was previously shown to enable standing and some voluntary control of leg movements. The two new studies provide the first evidence that epidural stimulation can enable overground stepping.

"Our group set out to do a proof-of-principle study to show that after a motor complete SCI, the human spinal cord retains intact locomotor circuitry below the level of injury and that this circuitry can be accessed

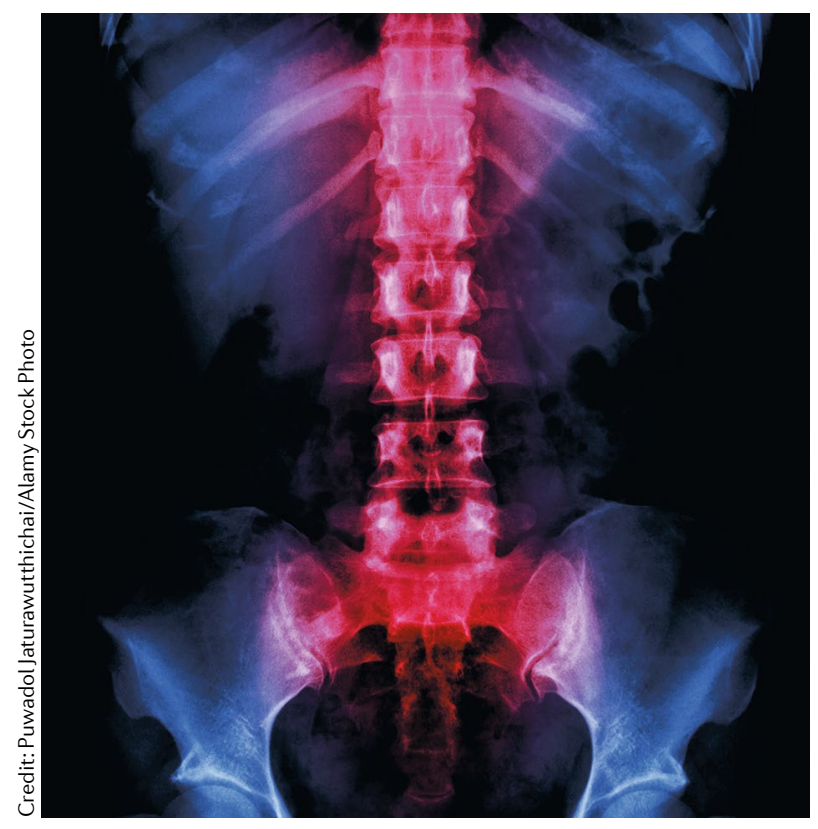

and retrained with the combination of locomotor training and epidural stimulation," explains Claudia Angeli, first author on the NEJM study.

Angeli and colleagues enrolled four participants with traumatic, motor complete damage in the cervical or thoracic spine, 2.5-3.3 years after initial injury. These individuals were unable to stand, walk or voluntarily move their legs.

Patients underwent intensive locomotor training for $2 \mathrm{~h}$ per day, 5 days per week for 8 to 9 weeks before implantation of an epidural stimulator. The team then tested combinations of stimulation patterns to find settings that caused activation of leg muscles to simulate walking motions.

The intervention enabled all participants to stand and sit independently. Two patients achieved overground walking when aided by electrical stimulation of the lower spinal cord. The other two participants could perform some treadmill stepping with body weight support but were not able to walk over ground.

The study showed that recovery of walking is feasible in some patients with spinal cord damage, even years after the initial injury. "Relearning the task of walking is possible in individuals classified as motor complete, who have been told that they will never be able to move their legs or walk again," Angeli remarks.

In the Nature Medicine study, a team led by Kristin Zhao and Kendall Lee at Mayo Clinic examined a patient with complete paraplegia resulting from an SCI that occurred 3 years before enrolment. The group had previously shown that epidural electrical stimulation in this individual restored the ability to stand and voluntarily perform stepping leg movements in a non-weight-bearing context.
In the new study, the patient underwent rehabilitation consisting of a range of different dynamic stimulation settings and motor tasks termed multimodal rehabilitation (MMR), during which epidural stimulation was optimized for the performance of each task. Over a 43-week course of MMR, the patient was able to step independently on a treadmill and could walk $>100 \mathrm{~m}$ over ground with the assistance of a wheeled walker and occasional balance assistance from trainers.

"The dynamic approach of MMR allowed the subject to stand and step; until now, this outcome was unattainable when using epidural electrical stimulation in humans with complete loss of leg function due to SCI," comments Zhao.

"These findings support the concept that the spinal networks are adaptable after severe injury and, with interventions such as spinal stimulation in combination with task-specific training, dysfunctional spinal circuitry can be modulated to regain some control of function," adds Lee.

The results are promising, but both groups agree that further investigation is needed before this technology can be translated for clinical use. "There is still a lot of work to be done," remarks Angeli. "First, we need to be able to reproduce these results in a larger number of individuals. Second, we need to improve the technology for this application to enable it to one day be moved into clinical practice."

Charlotte Ridler

ORIGINAL ARTICLES Angeli, C. A. et al. Recovery of over-ground walking after chronic motor complete spinal cord injury. N. Engl.J. Med. 379, 1244-1259 (2018) | Gill, M. L. et al. Neuromodulation of lumbosacral spinal networks enables independent stepping after complete paraplegia. Nat. Med. https://doi.org/10.1038/s41591-018-0175-7 (2018) 